\title{
The Residual Stress FEM Analysis of Pipeline Steel X70W
}

\section{Submerged-arc Welding}

\author{
YANG Zisen ${ }^{1, a}$, DI Quankang $2, b$, SUN Qisong ${ }^{3, c}$ \\ 1Shougang Research Institute of Technology, Shijingshan District, Beijing, China \\ ${ }^{2}$ Beijing Energy Steel Engineering Technical Research Center, Shijingshan District, Beijing, China \\ a309866845@qq.com, bdiquankang@mail.shougang.com.cn
}

Keywords: submerged-arc welding, crack, ANSYS, residual stress, welding speed

Abstract: The pipeline steel X70W submerged-arc welding was simulated by FEM using ANSYS, for the issue that the cracks were frequently found in the base metal when the pipeline steel was expanded holes after welding. And the residual stress was analyzed at three different welding speed through temperature field distribution and stress field simulating, a conclusion was obtained that the residual stress reduces as the welding speed reduces.

\section{Introduction}

The pipeline steel was mainly used for petroleum and natural gas pipeline, not only it's compression strength should be high, but also it's low-temperature toughness should be high and it's welding property should be excellent. The X70W pipeline steel was used widely, and the yield strength should be great than or equal to $485 \mathrm{MPa}$, the strength of extension should be great than or equal to 570MPa. The pipeline steel plate was used by submerged-arc welding, which is a welding method that the electric arc burns below the solder layer. Because the submerged-arc welding has advantages of welding stable quality, high productivity, no arc light and little smoke dust, so it was mainly used for pressure vessel, tabulation, box pile and beam column welding method during manufacturing.

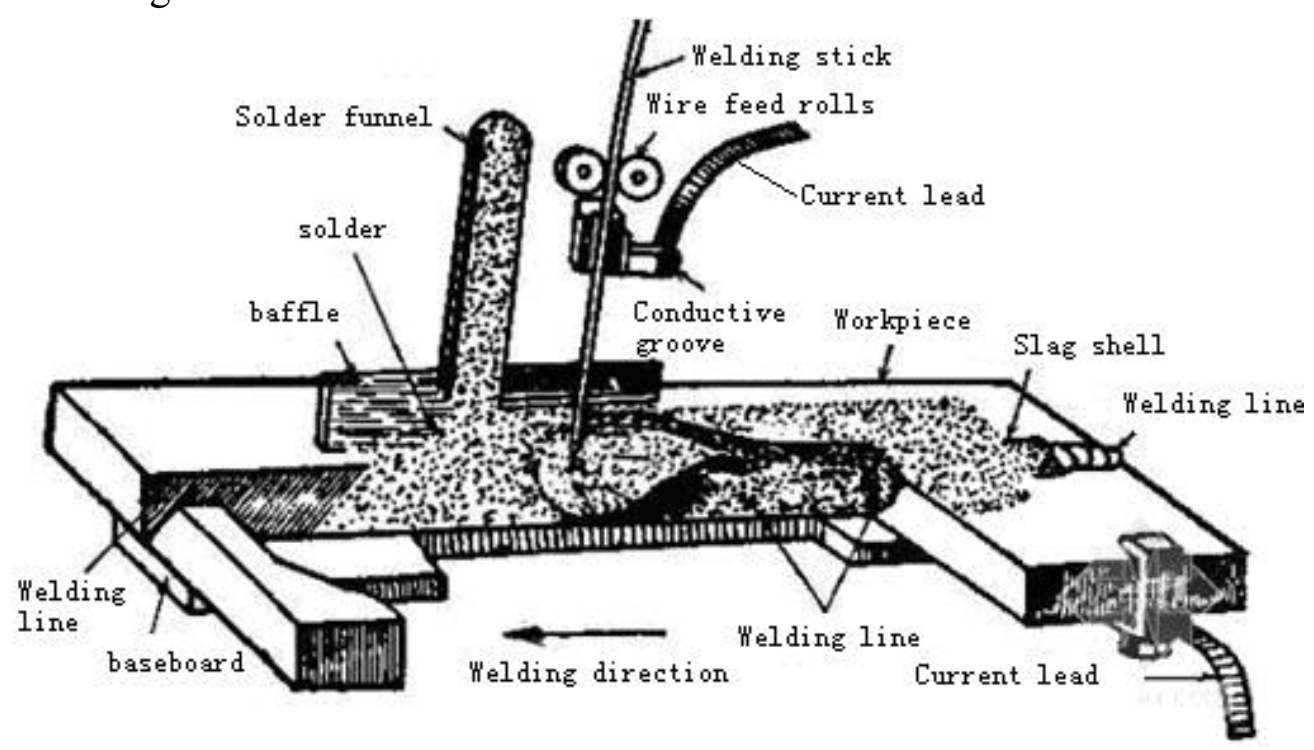

Fig1 The sketch map of submerged-arc welding

As the issue that the cracks were frequently found in the base metal when the pipeline steel $\mathrm{X} 70 \mathrm{~W}$ was spun over after welding, the relationship between the welding technological parameters 
and the residual stress was quantitative analyzed in this paper through submerged-arc welding temperature field distribution and stress field simulating, thus the cracks will be reduced through optimizing welding technological parameters.

\section{Residual stress theory and FEM model}

In the process of welding, as the metal around the weld joint area was heated quickly from indoor temperature to high melting temperature, then it was cooled gradually to indoor temperature, the mechanical property of the metal in and near the weld joint area takes a process from elastic to plasticity; while as the postwelding cooling goes on, the metal around the weld joint came to solidify and recover elastic. Therefore, the weld stress-stain field changes with transient temperature field, especially during the postwelding cooling, the solid-state phase transformation related to material will turn up volume expansion, which will trigger plastic deformation flow in microcell, even though the material is elastic. Mr Nagasaka found that because of postwelding phase transformation, the maximum stretch residual stress in lengthways direction reduces only in the area of weld joint and heat affected zone. Many researchers consider that solid-state phase transformation has an important influence on postwelding residual stress[1].

As low-carbon steel, because the transforming austenite into pearlite takes at high temperature which is higher than $600^{\circ} \mathrm{C}$, the material is almost nonelastic at this moment, the yield stress is very small, and the plasticity is quite well, so the transformation has little influence on the final residual stress. While as low content alloy high-strength steel and high alloy steel, the transformation often occurs below $600^{\circ} \mathrm{C}$, the yield stress and elasticity modulus of material recover quite well, so the transformation has a significance influence on the final residual stress[2].

\section{Temperature Model}

The analysis of welding temperature field belongs to typical transient heat conduction case. The equation of three-dimensional temperature field is as follows:

$$
\rho c \frac{\partial T}{\partial t}=\frac{\partial}{\partial x}\left(\lambda \frac{\partial T}{\partial x}\right)+\frac{\partial}{\partial y}\left(\lambda \frac{\partial T}{\partial y}\right)+\frac{\partial}{\partial z}\left(\lambda \frac{\partial T}{\partial z}\right)+Q
$$

In the equation, $\mathrm{Q}(\mathrm{x}, \mathrm{y}, \mathrm{z}, \mathrm{t})$ represents internal heat source intensity in the solving area $\mathrm{V} ; \mathrm{T}$ represents the function of temperature field; $\lambda$ represents heat conductivity coefficient; $\rho$ and $c$ respectively represent density and specific heat[3].

The equation above is generalized equation, specific solution conditions which are boundary conditions and initial values of differential equation, should be given in order to obtain specific solution. There are several kinds of boundary conditions of welding temperature field simulation as follows:

The first kind boundary condition, the temperature of the boundary was known:

$$
\lambda \frac{\partial T}{\partial x} n_{x}+\lambda \frac{\partial T}{\partial y} n_{y}+\lambda \frac{\partial T}{\partial z} n_{z}=T_{s}(x, y, z, t)
$$

The second kind boundary condition, the heat flux distribution of the boundary was known:

$$
\lambda \frac{\partial T}{\partial x} n_{x}+\lambda \frac{\partial T}{\partial y} n_{y}+\lambda \frac{\partial T}{\partial z} n_{z}=q_{x}(x, y, z, t)
$$


The third kind boundary condition, the heat exchange between object on the boundary and surrounding medium was known:

$$
\lambda \frac{\partial T}{\partial x} n_{x}+\lambda \frac{\partial T}{\partial y} n_{y}+\lambda \frac{\partial T}{\partial z} n_{z}=\beta\left(T_{a}-T_{s}\right)
$$

In the equation (2)-(4), $q_{x}$ represents external input heat source per unit area; $\beta$ represents surface heat transfer coefficient; $T_{a}$ represents object surface temperature; $T_{s}$ represents ambient temperature; $n_{x} 、 n_{y} 、 n_{z}$ respectively represent outer normal direction cosine[4].

\section{Stress field governing equation}

The force balance equation is:

$$
\sigma_{i j, j}=0
$$

$\sigma_{i j, j}$ represents stress component including thermal stress.

$$
\varepsilon^{T}{ }_{i j, j}=\alpha_{i j, j}\left(T-T_{0}\right) \delta i_{j, j}
$$

In the equation, $\varepsilon^{T}{ }_{i j, j}$ represents thermal strain tensor; $\alpha_{i j, j}$ represents coefficient of thermal expansion. $T_{0}$ represents reference temperature; $\delta i_{j, j}$ represents operator $\delta$.

Constitutive equation of stress and strain is:

$d \sigma_{i j}=D_{i j k l}\left(d \varepsilon_{k l}-d \varepsilon^{P}{ }_{k l}-d \varepsilon^{c}{ }_{k l}-d \varepsilon^{T}{ }_{k l}\right)$

In the equation: $D_{i j k l}$ represents constitutive tensor coefficient; $d \varepsilon_{k l}, d \varepsilon^{P}{ }_{k l}, d \varepsilon^{c}{ }_{k l}, d \varepsilon^{T}{ }_{k l}$ respectively represent total strain, plastic strain, creep strain and thermal strain[5,6].

\section{Boundary condition}

The cross section of pipeline steel $\mathrm{X} 70 \mathrm{~W}$ is $1016 \times 26.2 \mathrm{~mm}$, and the length of the steel plate is $12 \mathrm{~m}$, furthermore, the cross section of pipeline after welding is a ring of $\varphi 1016 \times 26.2 \mathrm{~mm}$. The $X$ groove was used in pipeline welding, as shown in the following figure2:

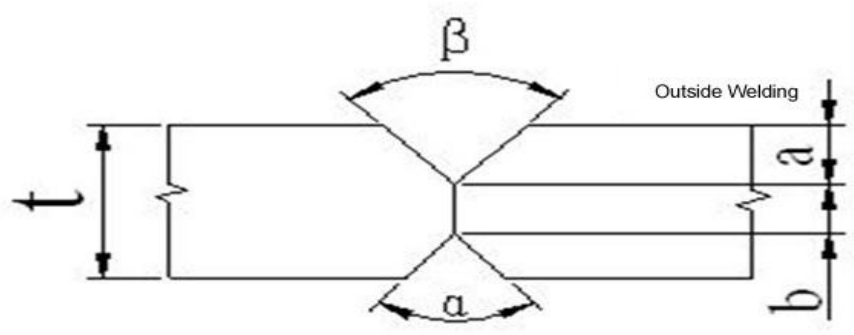

Fig2 The sketch map of $X$ welding groove $\left(\alpha: 70^{\circ}, \beta: 70^{\circ}\right.$;depth of outside the groove a:10mm; blunt edge b:9mm) 


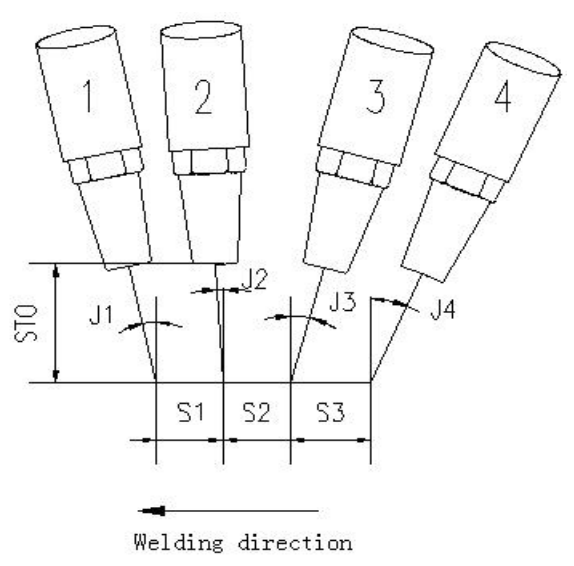

Fig3 The sketch map of welding torch $(\mathrm{S} 1=\mathrm{S} 2=\mathrm{S3}=19 \mathrm{~mm})$

The four welding torches were used for continuous submerged arc welding inside and outside the groove. Firstly, the internal groove was welded, then the outer groove was welded.

The two-dimensional model of $600 \times 26.4 \mathrm{~mm}$ was established along the cross section of the pipeline based on symmetry.

Table1 The chemical composition of base metal X70W \%

\begin{tabular}{c|c|c|c|c|c|c|c|c|c|c|c|c|c}
\hline $\mathrm{C}$ & $\mathrm{Si}$ & $\mathrm{Mn}$ & $\mathrm{P}$ & $\mathrm{S}$ & $\mathrm{Alt}$ & $\mathrm{Als}$ & $\mathrm{Ni}$ & $\mathrm{Cr}$ & $\mathrm{Cu}$ & $\mathrm{V}$ & $\mathrm{Nb}$ & $\mathrm{Mo}$ & $\mathrm{Ti}$ \\
\hline .07 & .15 & 1.55 & .01 & .003 & .038 & .036 & .181 & .058 & .139 & .018 & .06 & .214 & .015 \\
\hline
\end{tabular}

The liquidus temperature is $1518^{\circ} \mathrm{C}$, and the solidus temperature is $1479^{\circ} \mathrm{C}$. The weld pool temperature was taken the value of $1530^{\circ} \mathrm{C}$ in this paper.

Table3 Linear expansion coefficient /unit: $10^{-6}$

\begin{tabular}{c|c|c|c|c|c|c|c|c|c|c|c}
\hline Temperature $/{ }^{\circ} \mathrm{C}$ & $20-100$ & $20-200$ & $20-300$ & $20-400$ & $20-500$ & $20-600$ & $20-700$ & $20-800$ & $20-900$ & $20-1000$ & $700-1000$ \\
\hline Base metal & 11.66 & 12.32 & 13.02 & 13.65 & 14.22 & 14.64 & 15.01 & 14.67 & 13.14 & 13.35 & 9.5 \\
\hline Weld joint & 11.1 & 11.5 & 12.1 & 12.75 & 13.5 & 13.8 & 14 & 11.2 & 11.7 & & \\
\hline
\end{tabular}

The three different welding speed working conditions which are $1.14 \mathrm{~m} / \mathrm{min} 、 1.6 \mathrm{~m} / \mathrm{min}$ and $2.28 \mathrm{~m} / \mathrm{min}$, were calculated according to the actual welding process parameters in this paper.

\section{Calculation results of temperature field and residual stress field}

\section{Calculation results of temperature field}

From the results shown in Fig 4-5, the temperature of weld joint drops very quickly, and it drops to $71^{\circ} \mathrm{C}$ after welding $32 \mathrm{~s}$. The reasons are as follows: first of all, the weld joint and steel plate have huge difference in size; secondly, the heat conduction between high temperature molten pool area and low temperature region of base metal is quite fast; finally, the convection and heat 
radiation between the high temperature molten pool area and air have an important influence on the temperature drop. Through the temperature measurement of welding, the simulation results are in agreement with the measured results, which will ensure the accuracy of residual stress of the following calculation.

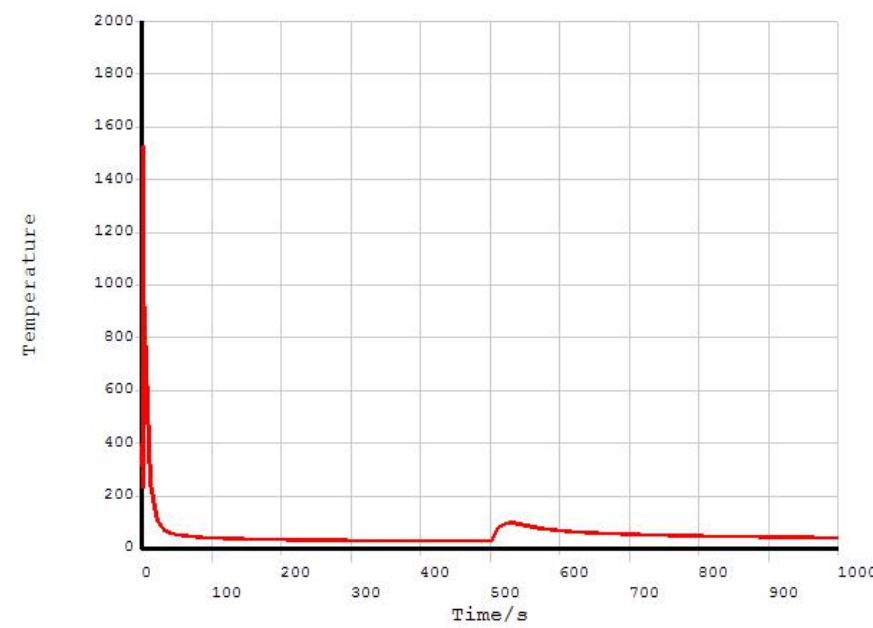

Fig4 Temperature change curve of a point in the groove welding zone
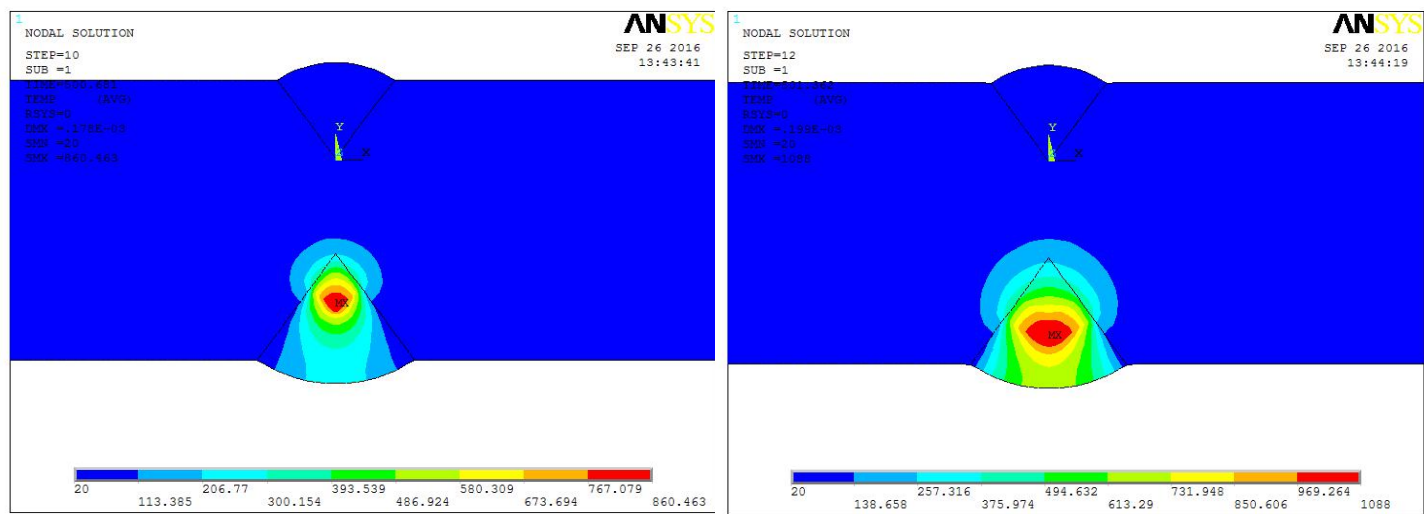

Before the second welding gun welding Before the third welding gun welding

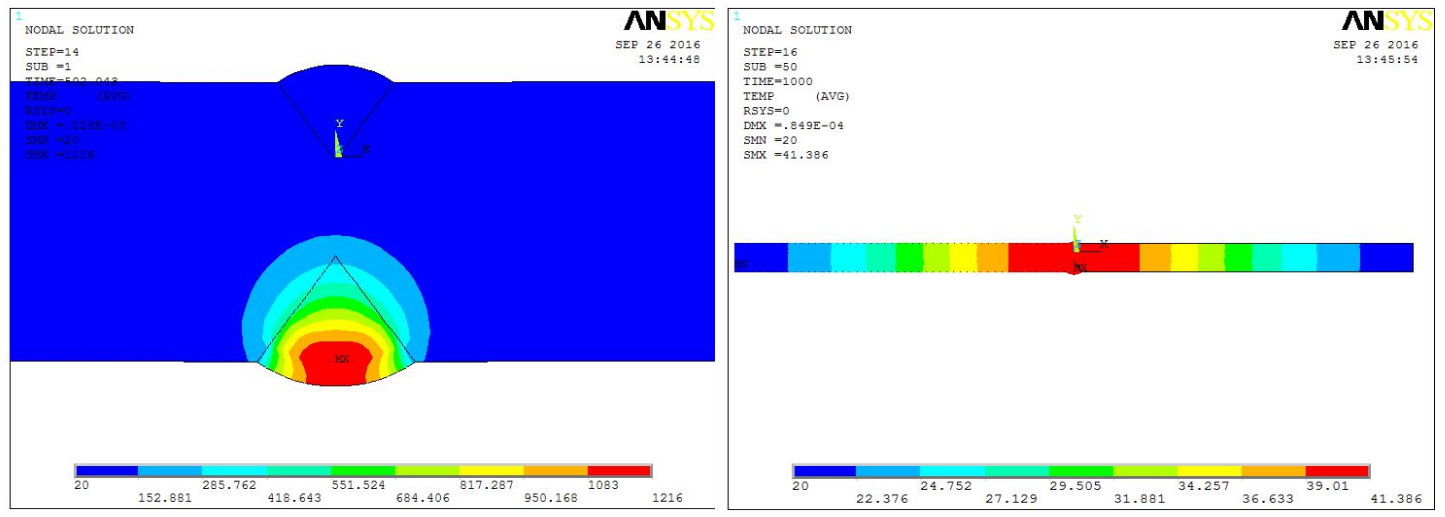

Before the fourth welding gun welding After welding 500s outside the groove Fig5 Temperature field distribution of welding model 


\section{Residual stress calculation results}
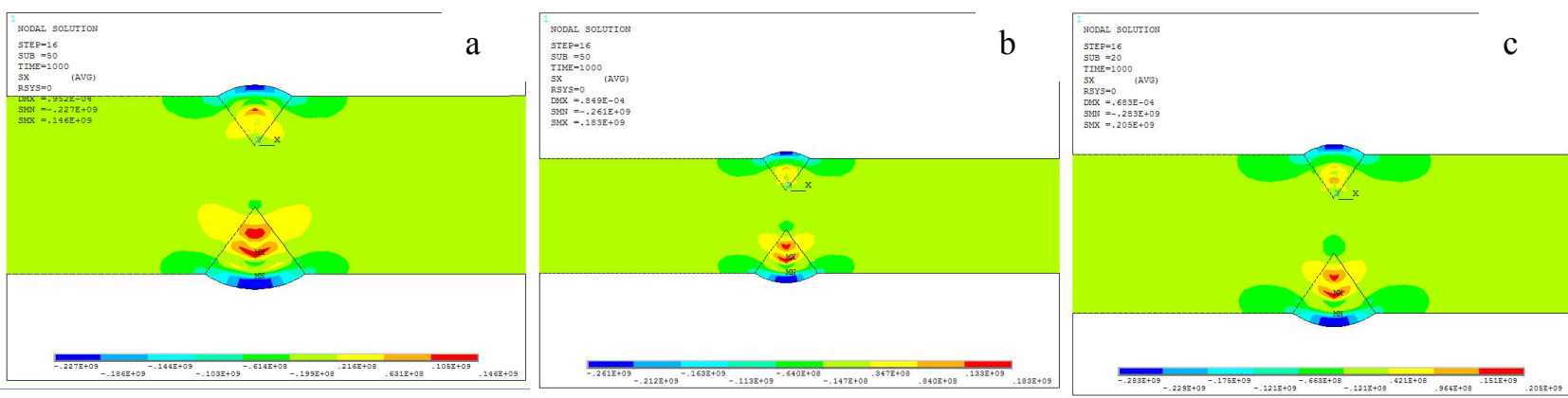

Fig6 Circumferential residual stress after welding
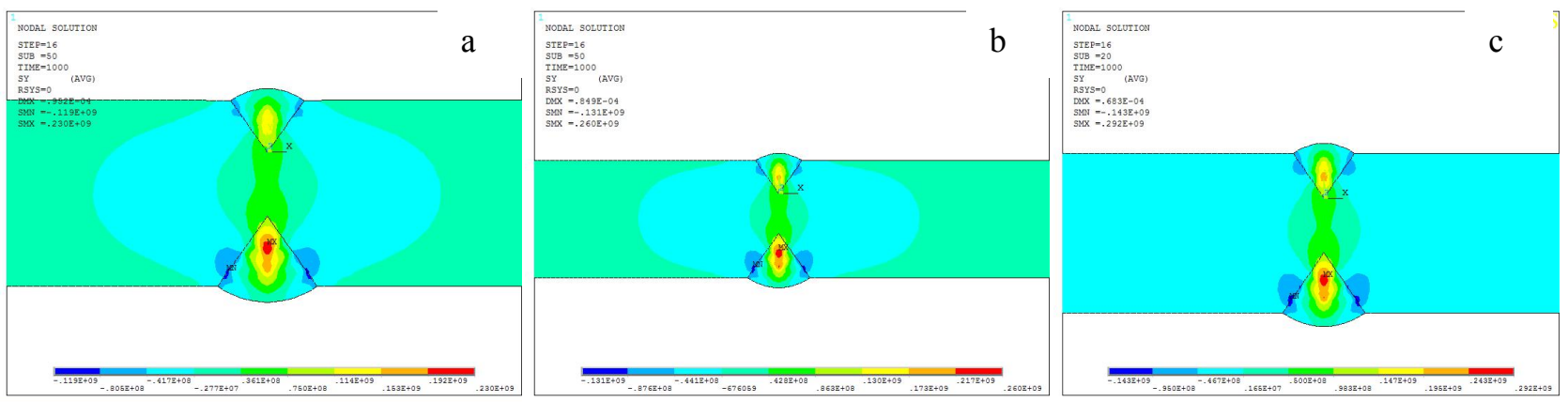

Fig7 Radial residual stress after welding
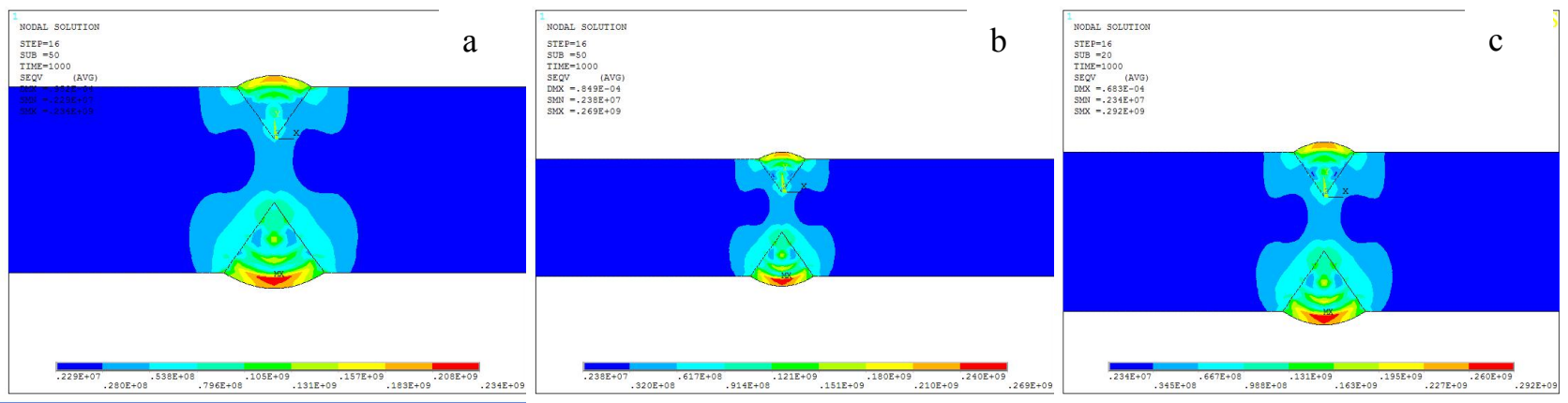

Fig8 Equivalent residual stress (VONMISES)

Welding speed: a: $1.14 \mathrm{~m} / \mathrm{min} ;$ b: $1.6 \mathrm{~m} / \mathrm{min} ;$ c: $2.28 \mathrm{~m} / \mathrm{min}$

By the above calculation results shown in Figure 6-8, the post-weld residual stress outside groove is lager than the inside groove when the welding speed is under the same condition; and the radial stress is significantly lager than the circumferential stress. Furthermore, the maximum circumferential residual stress occurs at the center of the welding seam, and the maximum residual stress of the three welding speeds are at $205 \mathrm{MPa}, 183 \mathrm{MPa}$ and $146 \mathrm{MPa}$. As the circumferential residual stress on the surface of welded joint is compressive, while the circumferential residual stress in the heart is tensile, so the heart is easy to crack when expanding holes.

According to the results of circumferential residual stress shown in Figure 6, the residual stress in the base metal around the weld joint which was welded by the second welding torch is lager than the other welding torches. Because of circumferential stress when expanding holes, so this position of the base metal is easy to crack, and the simulation position is consistent with the actual position where cracks occurs when expanding holes, so it verifies the accuracy of the calculation.

As shown in Figure 6 above, with the decrease of welding speed, the residual stress of the position of the base metal is decreased gradually, and it decreases from $42.1 \mathrm{MPa}$ to $21.6 \mathrm{MPa}$. In 
order to reduce the probability of crack generating in the base metal, the second welding torch process parameters need to be mainly controlled, therefore, the welding speed of the second welding torch should be reduced.

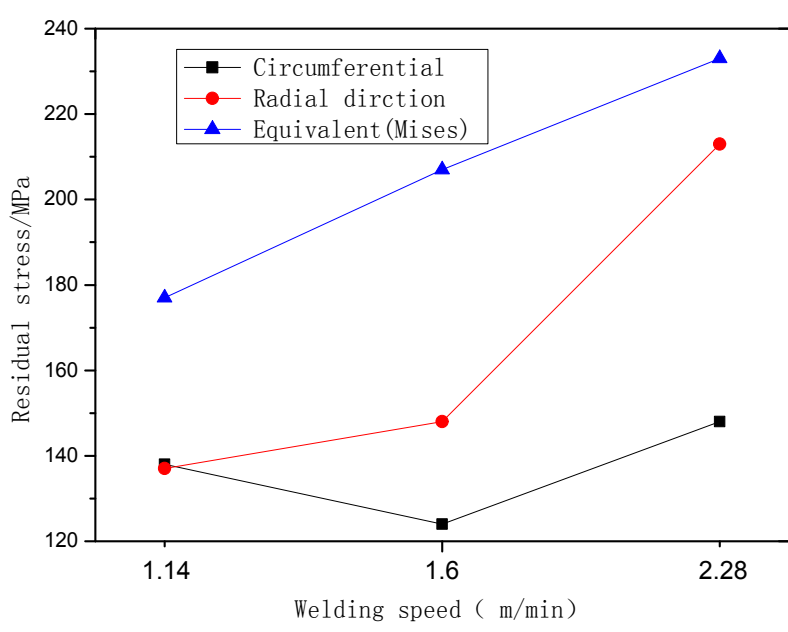

a. Inside groove

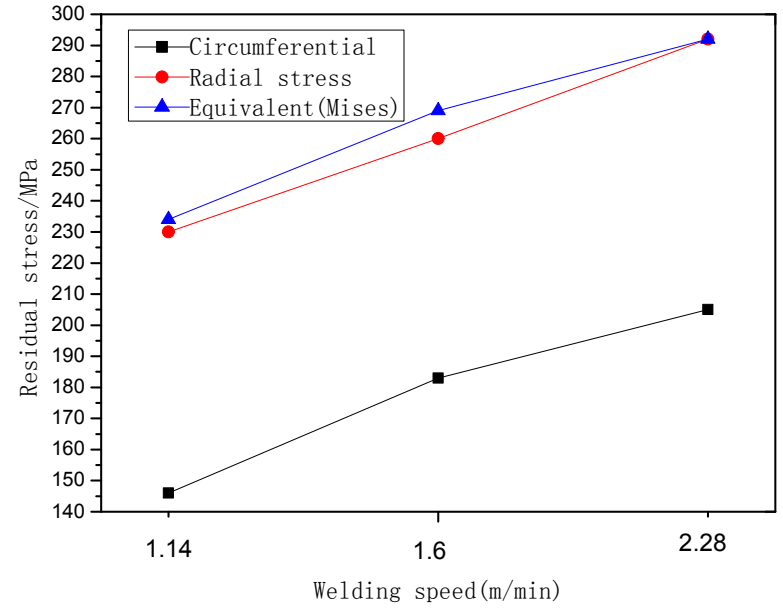

b. Outside groove

Fig9 The analysis on maximum residual stress inside and outside groove after welding

From the comparative analysis of residual stress in Figure 9, the residual stress increases gradually with the increase of welding speed in the welding process.

\section{Conclusion}

1. The residual stress outside the groove is greater than the inside one when the welding speed is in the same condition;

2. The maximum circumferential residual stress occurs at the centre of the weld, and it is tensioned in the centre where is easy to crack when expanding holes;

3. The maximum circumferential residual stress occurs in the base metal around the weld joint which was welded by the second welding torch, and this position is easy to crack when expanding holes;

4. The residual stress increases gradually with the increase of welding speed in the welding process.

\section{Reference}

[1] Shi Baoshan, He Kuanfang, He Hezhi Three dimensional dynamic simulation of temperature field of submerged arc surfacing, Experiment and research, 2009.03-0013-03

[2] Qi Shuai, Yan Chunyan, Shi Zhidan, Cui Xiaowei, Numerical simulation of longitudinal residual stress in three wire submerged arc welding of X80 pipeline steel, Welding machine, 2015 ( 04 ) 153-169.

[3] Li Rujuan, Li Mengsheng, Numerical simulation of the influence of the stress field of medium and heavy plate in welding process, Hot working process, 2006.35.73-75.

[4] Hu Shasha, LiXiao, NieJie, ShiWei, Numerical simulation of temperature field in submerged arc surfacing, Hot working process, 2013.02.210-212.

[5] Dong Junhui, Huo Lixing, Zhang Yufeng, Finite element analysis of welding residual stress of low carbon steel pipe, Welding 2000(12)11-15.

[6] Liang Xiaoyan, Luo Jinhua, Du Hanbin, Comparison of different welding heat sources in welding simulation based on ANSYS platform, Welding machine2003.03(33)29-32. 